denumerable ${ }^{u}$ ). Die beiden ersten Argumente gen lgten namentlich, um den Nachweis transzendenter 'Zahlen vom intuitionistischen Standpunkt aus wertlos zu machen, der nur Konstruktionen und keine reinen Existenzbeweise anerkennt.

Demgegentber ist hervorzuheben: der Nachueis transzendenter Zahlen durch das Diagonalverfahren erfolgt anf streng loonstruktivem Weg, und er ist sinnvoll und beweiskrë̈ftig für jeden (auch intuitionistischen) Standpunkt, der überhaupt einer der üblichen 'Zuordnungsregeln zwischen den algebraischen und den natürlichen Zahlen einen Sinn zuerkennt.

In der Tat: Bleiben wir bei der oben auseinandergesetzten Auffassung, wonach eine reelle Zahl gegeben - und zwar offenbar konstruktiv gegeben - ist, sobald eine Regel vorliegt, die ihre Dezimalbruchentwicklung so weit als gewunscht zu bilden gestattet! (Dor gelaufigeren Vorstellung und Ausdrucksweise wegen ist hier $n=10$ genommen. Ebenso beschränken wir uns der Bequemlichkeit halber auf reelle algebraische und transzendente Zahlen.) Es liege nun eine z. B. die Cantorsche - Zuordnungsregel zwischen den algebraischen und den naturlichen Zahlen vor; für jede algebraische Zahl, die durch die zugehörige irreduzible algebraische Gleichung und z. B. eine zusătzliche Größenkennzeichnung (unter den endlich vielen Wurzeln der Gleichung) gegeben sei, kann ihre Platznummer vermöge der Zuordnungsregel in endlich vielen Schritten ermittelt, andererseits ihre Dezimalbruchentwicklung bis zu jeder gew Unschter Stelle berechnet werden. So ist die $r^{\text {te }}$ Dezimalstelle der $r^{\text {ton }}$ algebraischen Zahl unserer Abzzhlung eine wohlbestimmte, berechenbare Ziffer $c_{r}$ zwischen 0 und 9, die Grenzen eingeschlossen. Wenn wir also, wie in Nr. 1, einen eindeutig bestimmten, Stelle fur Stelle berechenbaren Dezimalbruch durch eine Regel angeben, die die $r^{\text {te }}$ Ziffer $d_{r}$ als von $\boldsymbol{c}_{r}$ verschieden festsetzt, so erhalten wir eine reelle Zahl, die konstruktiv festgelegt und von jeder algebraischen Zahl verschieden ist. Wir haben damit eine ganz bestimmte transzendente Zahl kon struiert, wenn auch freilich die Ziffernfolge ihrer Dezimalbruchentwicklung nicht anschaulich übersehbar ist. Die in (D) (Beginn von Nr. 1) besprochene abzählbare Menge ist hier die Menge der algebraischen Zahlen.

1) Bridgman, loc. cit. p. 233. Gemeint ist offenbar, daß dio Mongo der algebraischen und der transzendenten Zablen abzlhlbar sei, oder jedenfulds Anderes zicht nachgewiesen werden kunne.

Jerusalem, Hebrew University, Einstein Institute of Mathematica.

\section{Contribution à la topologie des polytopes.}

Par

$$
\text { Karol B orsuk (Warszawa). }
$$

La formule bien connue due aux MM. S. Lef s chetz et H. H opf, concernant le nombre des points invariants des transformations continues ${ }^{1}$ ) implique que chaque polytope $P$ dont les nombres de Betti ${ }^{2}$ ) remplissent l'égalité

$$
\sum_{k=0}^{\infty} p_{k}(P)=1
$$

contient un point invariant par rapport à toute transformation continue en son sous-ensemble.

Les théorèmes sur la décomposition des polytopes que je vais démontrer dans cette Note nous permettrons de prouver que dans le cas des polytopes situés dans l'espace euclidien troisdimensionnel $R_{3}$ l'égalité (1) est non seulement suffisante, mais aussi nécessaire pour l'existence d'un point invariant par rapport à toute transformation continue en sous-ensemble.

Théorème 1. Chaque polytope ${ }^{3}$ ) connexe $P$ de dimension $\leqslant n$ se laisse décomposer en deux polytopes $P_{1}$ et $P_{2}$ dont le premier

1) Voir S. Lefschetz, Trans. Amer. Math. Soc. 28 (1926), 'p. $1-49$ et H. Hopf, Math. Zeitschr. 29 (1929), p. 493-524. Comp. aussi S. Lef gehetz, Topology, Now York 1930 , p. 359

2) Quant à la définition des nombres de Betti voir p. ex. le livre cité de M. Lefschetz, p. 34-35 (pour les polytopes) et p. 324-334 (pour les espaces compacts arbitraires). Le nombre de Betti de dimension $k$ d'un espace compact $E$ sera désigné ici par $p_{k}(E)$.

3) La thèse du théorème 1 serait en défaut pour les espaces un peu plus généraux que les polytopes, p. ex. pour les continus localement contractiles. Il existe, 
remplit les conditions: $p_{n}\left(P_{1}\right)=0 ; p_{l}\left(P_{1}\right)=p_{l}\left(I^{\prime}\right)$ pour $i \neq n$ et lo deuxieme est contractile dans soi $\left.{ }^{4}\right)$.

Dém onstration. Admettons que lo polytope l'est donné suus la forme d'un complexe gérmétrique $K$, c. ì d. décompusé on simplexes, ot désignons par $m$ le nombre de simplexos $n$-dimentionnols de $K$. Si dans $K$ il n'existe, sauf le cycle zóro, aucun cycle à $n$ dimensions aux coefficients rationnels (ce qui a lieu toujours dans le cas $m=0$ ), la thèse de notro théorème est remplie déjà par les polytopes $P_{1}=P$ et $P_{2}$ ne contenant qu'un seul point étant sommet de $P$. Nous procédons maintenant par induction en admettant que $m=m_{0}+1$ et qu'il existe dans le cas $m \leqslant m_{0}$ les polytopes $P_{\mathbf{1}}$ et $P_{2}$ remplissant, outre la thèse de notre théorème, la condition que $P_{1}$ contienne tous les simplexes de dimension $<n$ de $K$. Nous pouvons admettre en outre qu'il existe duns $K$ un cyclo $n$-dimensionnel $C^{(n)}$ aux coefficients rationnels ot un simplexe géométrique $n$-dimensionnel $\Delta$ de $K$ qui figure (après lui avoir donno uno orientation) dans $C^{(n)}$ avec un coefficient différent de 0 .

Désignons par $P^{\prime}$ le polytope qu'on obtient de $P$ en enlevant l'intérieur du simplexe $\Delta$. Le polytope $P^{\prime}$ ainsi défini est donné sous la forme d'un sous-complexe $K^{\prime}$ de $K$ contenant $m_{0}$ simplexes de dimension $n$ et tous les simplexes de dimension $<n$ de $K$.

Nous allons maintenant démontrer que $P^{\prime}$ remplit on outre la condition $p_{t}\left(P^{\prime}\right)=p_{i}(P)$ pour $i \neq n$. Tous les cycles dans $K$ à $i<n$ dimensions étant des cycles dans $K^{\prime} \subset K$, il suffit à ce but de prouver que pour chaque complexe (algébrique, aux coefficients rationnels) $Q$ de $K$ il existe dans $K^{\prime}$ un complexe (algébrique) $Q^{\prime}$ dont la frontière $\dot{Q}^{\prime}$ est égale à la frontière $\dot{Q}$ de $Q$. En tenant compte du fait que la frontière du simplexe $\Delta$ (dont on a donné une orientation) se laisse exprimer à l'aide de la relation $\dot{C}^{(n)}=0$ comme

notamment, même dans $R_{3}$, des continus localement contractiles ayant le promier nombre de Betti égal à 0 et le deuxième égal à 1, qui ne se laissent pas décomposer en un nombre fini (ou mềme dénombrable) de ses vrais sous-ensembles fermés ayant poux le premier nombre de Betti 0. On obtient un tel exemplo par une lépère modification de l'exemple qui se trouve dans la note de M. S. Mazurkiewioz ot do moi, C. R. 199 (1934), p. 110-112

9) L'espace $E$ est dit contractile dans soi, lor'squ'il existe une fonetion $p(x, t)$ définie pour $x \in E$ et $0 \leqslant t \leqslant 1$, continue pour les deax variables $x$ et $t$ it la fois et remplissant les conditions; $\varphi(x, 0)=x ; \varphi(x, t) \in E$ et $\varphi(x, 1)=$ const, quels que soient $x \in E$ et $0 \leqslant t \leqslant 1$ la frontière d'un complexe (algébrique) $R$ situé dans $K^{\prime}$, on obtient le complexe demandé $Q^{\prime}$ en remplaçant dans $Q$ le simplexe $\Delta$ (orienté) par le complexe $R$.

L'égalité $p_{i}\left(P^{\prime}\right)=p_{i}(P)$ pour $i \neq n$ étant ainsi démontrée, il exisle par hypothèse une décomposition de $P^{\prime}$ en deux polytopes $P^{\prime}$ et $P_{2}^{\prime}$, dont le premier remplit les conditions

$$
p_{n}\left(P_{1}^{\prime}\right)=0, \quad p_{i}\left(P_{1}^{\prime}\right)=p_{i}(P) \quad \text { pour } \quad i \neq n,
$$

en contenant, en outre, tous les simplexes de dimension $<n$ du complexe $K^{\prime}$, donc aussi tous les simplexes de dimension $<n$ du complexe $K$, et le deuxième et contractile dans soi.

Envisageons maintenant un petit simplexe $n$-dimensionnel $\Delta_{0}$ situé tout entier dans l'intérieur du simplexe $\Delta$. Désignons par $T$ le polytope qu'on obtient de $\Delta$ en enlevant l'intérieur du simplexe $\Delta_{0}$ et par $L$ une ligne simple brisée contenue dans $P$ dont les extrémités $a$ et $b$ appartiennent l'une à $\Delta_{0}$ et l'autre à $P_{2}^{\prime}$ et dont tous les autres points sont placés en dehors de $P_{2}^{\prime}+\Delta_{0}$.

Posons :

$$
P_{1}=P_{1}^{\prime}+T ; \quad P_{2}=P_{2}^{\prime}+L+\Delta_{0} .
$$

On peut, bien entendu, contracter $\Delta_{0}$ dans soi vers le point $a$ par une déformation qui laisse ce point immobile et ensuite contracter la ligne brisée $L$ vers $b$, en laissant $b$ immobile. Par cette opération $P_{2}$ sera déformé en $P_{2}^{\prime}$, c. à d. en un polytope contractile dans soi. Les formules (3) entraînent en outre que $P_{1}+P_{2}=P$ et que $P_{1}$ contient tous les simplexes de dimension $<n$ de $K$. Pour achever notre démonstration il suffit donc, d'après les formules (2), de prouver que les nombres de Betti du polytope $P_{1}$ sont égaux à ceux du polytope $P_{1}^{\prime}$. A ce but, désignons par $c$ le centre de gravité de $\Delta_{0}$ et par $x^{*}$, pour tout $x \in T$, la projection de $x$ du centre $c$ sur la frontière $\dot{\Delta}$ du simplexe $\Delta$. Posons, pour $0 \leqslant t \leqslant 1$ :

$\varphi(x, t)=$ point $\mathrm{du}$ segment $\bar{x} \bar{x}^{*}$ remplissant la condition

$$
\varrho(x, \varphi(x, t))=t \cdot \varrho\left(x, x^{*}\right),
$$

si $x \in T$ et

$$
\varphi(x, t)=x
$$

si $x \in P_{1}^{\prime}$. 
Il est évident que la fonction $\varphi(x, t)$ ainsi définie fait une déformation continue de l'ensemble $P_{1}$ dans soi, en le transformant pour $t=1$ en $P_{1}^{\prime}+\dot{\Delta}=P_{1}^{\prime}$ (puisque $P_{1}^{\prime}$ contient tous les simplexes $(n-1)$-dimensionnels de $K$ ) et en laissant tous les points de $P_{1}^{\prime}$ immobiles pour tout $0 \leqslant t \leqslant 1$. Il en résulte ") que tous les nombres de Betti de $P_{1}$ sont égaux à cenx de $P_{1}^{\prime}$, c. q. f. d.

Remarque. Dans le eas ou $n=2$ ot $p_{1}(P)=0$, los nombres do $13 \theta t$ ti du polytope $P_{1}$ de la thèse du théorème 1 sont égaux à ceax du polytopo $P_{a}$ contractile dans soi. On ne peut pas cependant affirmer qu'il oxiste dans co cas uno décomposition de $P$ en deux polytopes contractiles dans soi, même si le polytope $P$ est acyclique en dimension 1. (c. à d. que tous les cyoles 1-dimonsionnels aitroés daxis $P$ aux coefficients quelconques sont les frontiòres des complexes algébriques situés dans $P$ ). Ainsi p. ox. lo polytope $P$ (ju'on obtiont de l'espace bion connu do

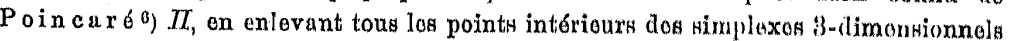
d'une décomposition simpliciale do $I I$, ont acyclique on dimension 1 , mais son groupe fondamental contient plus d'un élément. Il n'existe done ") aucune décomposition de $P$ même on deux polytopes contractiles dane $P$.

Théorème 2. La thise du théorìme 1 reste valable pour tous les polytopes connexes situés dans un espace euclidien ì $(n+1)$-dimensions $R_{n+1}$.

Démonstration ${ }^{8}$ ). Admettons que le polytope $P$ est donné sous la furme d'un complexe géométrique $K$ à $m$ simplexes de dimension $n+1$. La thèse du théorème 2 étant vraie (d'après lo théorème 1) dans le cas $m=0$, nous pouvons procéder par induction en admettant que $m=m_{0}+1$ et que pour $m \leqslant m_{0}$ il existe la décomposition en question. Il résulte de nos hypothèses l'existence d'un simplexe $(n+1)$-dimensionnel $\Delta^{(n+1)}$ ayant une face $n$-dimensionnelle $\triangle^{(n)}$ contenue dans la frontière de $P$ (frontière relative a $R_{\text {ats }}$ ). On constate sans peine que pour un point $a \in R_{n+1}-P$ situé suffisamment près du centre de gravité du simplexe $\Delta^{(n)}$, chaque demidroite $\overrightarrow{a x}$, oì $x \in \Delta^{(n+1)}$, coupe $\Delta^{(n+1)}$ le long d'un segment $L(x)$

5) Fund. Math. 21 (1933), p. 91-92.

6) On comprend par un espace de Poincaré une variété à trois dimensions dont toutes les propriétés de l'homologie coìncident avec celles d'une surface sphérique exclidienne à trois dimensions, mais dont le groupe fondamentel contient plas d'un élément.

7) Cf. C. R. 198 (1934), p. 1732, th. V.

9) La même démonstration reste valable dans lo cas pluns gónóral, ở, au liou de supposer que $P C R_{n+1}$, on suppose seulement que lo polytope $P$ est un vaai sous-ensemble d'une pseudovariété queleonque ì $n+1$ dimensions. (qui peut se réduire à un seul point) dont une extrémité est située sur $\Delta^{(n)}$ et l'autre, que nous désignons par $x^{*}$, sur les autres faces $n$-dimensionnelles de $\Delta^{(n+1)}$ et que $L(x)$ coüncide avec la partie commune du segment $\overline{a x}^{*}$ et du polytope $P$. Pour deux points $x_{1}$ et $x_{2}$ de $\Delta^{(n+1)}$ les segments $L\left(x_{1}\right)$ et $L\left(x_{2}\right)$ sont, bien entendu, identiques ou disjoints. Posons, pour tout $0 \leqslant t \leqslant 1$ :

$\psi(x, t)=$ point du segment $L(x)$ remplissant l'égalité

$$
\varrho(x, \psi(x, t))=t \cdot \varrho\left(x, x^{*}\right)
$$

si $x \in \Delta^{(n+1)}$ et

si $x \in P-\Delta^{(n+1)}$.

$$
\psi(x, t)=x,
$$

La fonction $\psi$ ainsi définie constitue, comme on vérifie facilement, une déformation continue du polytope $P$ dans soi, en le rétractant ${ }^{9}$ ) pour $t=1$ en polytope $P^{\prime}$, qu'on obtient de $P$ en enlevant les points intérieurs des simplexes $\Delta^{(n+1)}$ et $\Delta^{(n)}$. Il en résulte ${ }^{5}$ ) que les nombres de Betti de $P^{\prime}$ sont égaux à ceux de $P$. Par conséquent, le, polytope $P^{\prime}$ satisfait aux prémisses de notre théorème en admettant par sa définition, une décomposition simpliciale renfermant $m_{0}$ simplexes de dimension $n+1$. En vertu de notre hypothèse, il existe une décomposition de $P^{\prime}$ en deux polytopes $P_{1}^{\prime}$ et $P_{2}^{\prime}$ dont le premier remplit les conditions (2) et le deuxième est contractile dans soi.

Désignons maintenant par $P_{i}$ (où $i=1,2$ ) la somme du polytope $P_{i}^{\prime}$ et de tous les segments $L(x)$ tels que $x^{*} \in P_{i}^{\prime}$. L'ensemble $P_{i}^{\prime} \cdot \Delta^{(n+1)}$ étant - comme partie commune de deux polytopes - un polytope, la somme des segments en question, et parsuite l'ensemble $P_{l}$ tout entier, sont aussi des polytopes. En outre, il est évident que la fonction $\psi(x, t)$ (n'envisagée que pour $x \in P_{l}$ et $0 \leqslant t \leqslant 1$ ) constitue une déformation continue du polytope $P_{i}$ dans soi et que $\psi(x, 1)$ est une fonction rétractante $\left.{ }^{9}\right) P_{i}$ en $P_{i}^{\prime}$. Par conséquent ${ }^{5}$ ) les nombres de Betti de $P_{1}$ sont éganx à ceux de $P_{1}^{\prime}$ d'où, selon (2):

$$
p_{n}\left(P_{1}\right)=0, \quad p_{i}\left(P_{1}\right)=p_{i}(P) \quad \text { pour } \quad i \neq n \text {. }
$$

9) Un sous-ensemble $A$ d'un espace $E$ s'appelle rétracte de $M$, lorsqu'il existe une fonction $f$ (fonction rétractante $E$ en $A$ ) transformant $E$ en $A$ d'une manière continue et telle que $f^{\prime}(x)=x$ pour tout $x \in A$. Si pour tout espace $E \supset A$ l'ensemble $A$ est un rétracto de $E$, alors $A$ s'appelle rétracte absolu. 
Le polytope $P_{2}$ étant - comme défurmable dans soi en l'enm semble $P_{2}^{\prime}$ - contractile dans soi, on en déduit que la décomposition de $P$ en $P_{1}$ et $P_{2}$ est de la forme demandée, c. q. f. d.

En tenant compte du théorème ${ }^{10}$ ), d'après lequel torut polytope $P$ situé dans l'espace euclidien 3-dimensionnel $R_{8}$ et remplissant la condition $p_{2}(P)=p_{1}(P)=p_{0}\left(P^{P}\right)-1=0$ est contractile dans soi, on parvient du théorème 2 au

Corollaire. Chaque polytope connexe situé dans $R_{\mathrm{B}}$ et dont le premier nombre de Betti disparait se laisse dicomposer on deux polytopes contractiles dans soi.

Pour prouver le dernier théorèmo do cette note, nous démontrerons d'abord le simple lemme suivant

Lemme. Toute fonction continue $f$ transformant la partie com mune de deux rétractes absolus $\left.{ }^{9}\right) A$ et $B$ en son sous-ensemble se laisse pralonger sur l'ensemble $A+B$ tout entier de fason que la fonction prolongée $f^{\prime}$ est continue, ses valeurs appartiennent d $A+B$ et qu'elle n'admet aucun point invariant dans l'ensemble $(A+B)-A \cdot B$.

Démonstration. Les ensembles $A$ et $B$ étant des rétractes absolus, il existe ${ }^{11}$ ) un prolongement continu $f_{1}$ de $f$ sur l'ensemble $A$ dont les valeurs appartiennent à $B$ et un prolongement continu $f$ de $f$ sur l'ensemble $B$ dont les valeurs appartiennent a $A$. La fonction $f^{\prime}$ définie par les formules

$$
\begin{aligned}
& f^{\prime}(x)=f_{1}(x) \text { pour tout } x \in A, \\
& f^{\prime}(x)=f_{2}(x) \text { pour tout } x \in B
\end{aligned}
$$

remplit, comme on vérifie aisément, la thèse de notre lemme.

Theorème 3. Pour qu'un polytope $P$ situé dans l'espace euclidien 3-dimensionnel se laisse transformer en son sous-ensemble d'une fasion continue et sans points invariants, il faut et il suffit qu'il remplisse la condition $\sum_{k=0}^{\infty} p_{k}(P) \geqslant 2$.

Démonstration. La nécessité de la condition étant une conséquence immédiate de la formule générale de MM. L e f s ohetz

10) Monatsh, f. Math. u Phys, 41 (198x), p. 78.

11) Fand. Math. 17 (1931), p. 157. et $\mathrm{H}$ op ${ }^{12}$ ), il ne reste qu'à démontrer sa suffisance. En tenant compte du fait évident que chaque ensemble non connexe se laisse transformer sans points invariants en son sous-ensemble par une fonction continue, et du théorème ${ }^{18}$ ) d'après lequel cela a lieu anssi pour tous les continus péaniens dont le premier nombre de Betti est positif, il reste seulement à prouver qu'il existe pour un polytope $P C R_{3}$ remplissant la condition

$$
p_{0}(P)=p_{1}(P)+1=1 \leqslant p_{2}(P)
$$

une fonction continue transformant $P$ en un sous-ensemble sans points invariants. D'après le corollaire du théorème 2 il existe une décomposition de la forme $P=P_{1}+P_{2}$, où $P_{1}$ et $P_{2}$ sunt des polytopes contractiles dans soi. En tenaut compte de la formule bien connue ${ }^{14}$ ), concernant la relation entre les propriétés de la homologie des polytopes $P_{1}, P_{2}, P_{1}+P_{2}$ et $P_{1} \cdot P_{2}$, on parvient à la relation $p_{1}\left(P_{1} \cdot P_{2}\right)=p_{2}(P) \geqslant 1$. Il en résulte $\left.{ }^{13}\right)$ l'existence d'une fonction continue $f$ transformant $P_{1} \cdot P_{2}$ en sous-ensemble sans points invariants. Les polytopes $P_{1}$ et $P_{2}$ étant contractiles dans soi, donc des rétractes absolus ${ }^{16}$ ), on conclut d'après notre lemme que la fonction $f$ se laisse prolonger sur l'ensemble $P=P_{1}+P_{2}$ de façon que le prolongement $f^{\prime}$ transforme l'ensemble $P$ en sousensemble de $P$ d'une manière continue et sans points invariants.

La démonstration du théorème 3 est ainsi terminée.

Il est à remarquer que la thèse du théorème 3 serait en défaut pour des polytopes, quelconques (non situés dans $R_{3}$ ). Ainsi par

12) Il est à remarquer que la simple conséquence de la formule de MM. Lefs s che ta et Hopf, la seule que nous utilisons dans cette Note, se laisse obtenir d'une façon élémentaire indépendamment de la formule générale. Dans le cas notamment où $P$ est un polytope situé dans $R_{\mathrm{s}}$, la condition $p_{2}\left(P^{\prime}\right)=p_{1}(P)=p_{0}(P)-1=0$ entraîne que $P$ est an rétracte absolu (voir Monatsh. $f$. Math. u. Phys. 41 (1934), p. 73) et parsuite (voir Fund. Math. 17 (1931), p. 163) qu'il existe un point invariant par rapport à toute transformation continue de $P$ en son sous-ensemble.

13) Fund. Math. 20 (1933), p. 231.

14) Voir W. Mayer, Monatsh. f. Math. u. Phys. 36 (1929), p. 40, formule (96). Dans le cas particulier où les polytopes $P_{1}$ et $P_{2}$ sont tels que tous les cycles de dimension $\geqslant 1$ situés dans $P_{l}$ (où $i=1,2$ ) sont homologues à zéro dans $P_{i}$, la formule mentionnée prend la forme bien simple: $p_{k+1}\left(P_{1}+P_{\mathrm{a}}\right)=p_{k}\left(F_{1} \cdot P_{2}\right)$, pour $k=1,2, \ldots$

15) Fund. Math. 19 (1932), p. 229. 
exemple ${ }^{16}$ ) le plan projectif complexe $K_{2}$ (étant une variété à 4 dimensions) remplit la condition $\underset{k \text { mo } 0}{\sum_{k}} p_{k}\left(K_{q}\right)=3$, bien qu'il ne se laisse pas transformer en son sous-ensemble d'une manière continue et sans points invariants ${ }^{17}$ ).

La question suivante reste ouverte:

La thèse du théorème 3 reste-t-elle vraie pour tous les continus localement contractiles ${ }^{18}$ ) situés dans $R_{\mathrm{g}}$ ?

16) C'est M. H. Hopf qui m'a indiqué cot exemple.

17) Voir H. Hopf, Journ. f. d. x. a. a. Math. 168 (1980), p. 85.

18) La question analogue dans le domaine des continus púaniens arbitraires situós dans $R_{3}$ est rósoluo dans lo sens nógntif, ear il existe dans $R_{n}$ un continu péanien dont les nombres de Betti sont éguux ì cusux du simplexo ot qui so laisso transformor topologiquement on lui-même sans pointa invariants. Voir liund. Math. 24 (1934), p. 52. La question de l'oxistenco dans $R_{11}$ l'un eontỉnu pónion $C_{\text {qui }}$ contienne un point invariant par rapport at toute trunfformation continue on fom sous-onsemble et remplisse la condition $\sum_{k=0}^{\infty} p_{k}(C) \geq 2$ rostite copendant onvorte.

Wiązowszczyzna, Nö̈l 1934 .

\section{Sur la généralisation des conditions de Cauchy-Riemann.}

Par

\section{Mench off (Moscou).}

§ 1. Soit $f(z)=u(x, y)+i v(x, y)$ une fonction continue d'une variable complexe $z=x+i y$ définie dans un domaine ouvert $D$, où $x, y, u(x, y)$ et $v(x, y)$ sont réels. Supposons qu'en chaque point $z$, intérieur au dumaine $D$, les dérivées partielles $\frac{\partial u}{\partial x}, \frac{\partial u}{\partial y}, \frac{\partial v}{\partial x}$ et $\frac{\partial v}{\partial y}$ existent et vérifient les relations

$$
\frac{\partial u}{\partial x}=\frac{\partial v}{\partial y}, \quad \frac{\partial u}{\partial y}=-\frac{\partial v}{\partial x} .
$$

Dans ces conditions la fonction $f(z)$ est holomorphe à l'intérieur du domaine $D^{1}$ ).

Nous pouvons énoncer ce théorème d'une autre manière. Soit $d$ une droite infinie située dans le plan du domaine $D$ et passant par un point $z$. Désignons par $\lim (d) A(z, h)$ la limite de la quantité $\mathcal{A}(z, h)$, lorsque $h$ tend vers zéro de telle façon que le point $z+h$ reste toujours sur la droite $d$. Nous pouvons énoncer le théorème précédent de la manière suivante.

Supposons qu'en chaque point $z$, intérieur au domaine $D$, les limites

$$
\lim _{h \rightarrow 0}(d) \frac{f(z+h)-f(z)}{h}
$$

1) Moutel, Sur les différentielles totales et les fonctions monogènes, C. $\mathbf{R}_{\mathrm{.}}$ Acad. Sci. Paris, pp. 1820-1822 (1913).

L o oman, Über eine Erweiterung des Cauchy-Goursatschen Integralsatzes, Nieuw. Arch. Wiskde, pp. 97-108, (1923).

Saks, Théorie de l'intégrale, Monografje Matematyczne II, pp. 242-246, Warszawa, (1933). 